\title{
Guest-editorial
}

\section{Special issue on Hybrid Fuzzy Models}

\author{
José M. Benítez ${ }^{\mathrm{a}, *}$, Salvador García ${ }^{\mathrm{b}}$, Santi Caballéc and Ángel A. Juan ${ }^{\mathrm{c}}$ \\ ${ }^{a}$ Department of Computer Science and Artificial Intelligence, Universidad de Granada, Granada, Spain \\ ${ }^{\mathrm{b}}$ Department of Computer Science, University of Jaén, Jaén, Spain \\ ${ }^{\mathrm{c}}$ Open University of Catalonia, Barecelona, Spain
}

This special issue is composed of five papers addressing different topics in current developments of Hybrid Fuzzy Models. The issue originated from presentations at the 8th International Conference on Hybrid Intelligent Systems that was held in Barcelona, Spain, September 10-12th, 2008. Selected contributions were extended and went through a blind-fold peer review process.

In this special issue several topics on the hybridization of fuzzy systems with other techniques are covered. Most of the papers have classification problems as their target application, but regression is also considered.

The first paper, "Comparison of Data Driven Models for the Validation of Residentials Premises using the KEEL Tool", develops a thorough empirical study of the performance of twelve different models applied to problem of computing the market value of a property. This is a very important problem due to the scope of people that it affects. The paper employs KEEL as an experimentation tools and highlights the good features the system offers for this purpose.

The second article, "A Bibliometric Study about the Research based on Hybridating the Fuzzy Logic Field and the Other Computational Intelligent Techniques: A Visual Approach", is quite helpful for the researchers in the area. It draws clear conclusions about the leading topics in the hybridization of Fuzzy Logic with other techniques included within Soft Computing. In partic- ular, a description of these topics evolution is provided as well as remarking the most active journals and subject categories.

In the third contribution, "Fuzzy-CCM: a contextsensitive approach to fuzzy modeling", a new fuzzy modeling technique based on conditional fuzzy algorithm is proposed. The method is mainly aimed at improving the interpretability of the rule bases while trying to keep a high accuracy.

The fourth paper, "Comparing Two Genetic Overproduce-and-choose Strategies for Fuzzy Rule-based Multiclassification Systems Generated by Bagging and $\mathrm{Mu}-$ tual Information-based Feature Selection", offers an extension of a former method to build fuzzy rule-based systems. The authors develop on two multicriteria genetic algorithms to find a good multiclassifier with an appropriate accuracy-complexity trade-off.

In the fifth paper, "A goal-related distance function on fuzzy partitions and its use in nearest neighbor classification", the well-known nearest neighbor classification algorithm is enriched through the use of a new distance. This distance allows the classifier to adapt through its learning experience.

Finally, as Guest Editors of this special issue, we would like to thank all the authors for their contributions and the referees for their outstanding cooperation and constructive comments.

\footnotetext{
*Corresponding author. E-mail: j.m.benitez@decsai.ugr.es.
} 